\title{
Flexible Ammonia Sensor Based on Polyaniline/Carbon Black Composites Operating at Elevated Temperatures
}

\author{
Ehsan Danesh, Krishna C. Persaud \\ School of Chemical Engineering \& Analytical Science, \\ The University of Manchester, Manchester, M13 9PL, UK \\ krishna.persaud@manchester.ac.uk
}

\begin{abstract}
A highly sensitive ammonia sensor has been fabricated by depositing solution processable polyaniline/carbon black conductive composites on to flexible polyimide substrates with gold interdigitated electrodes. This was tested toward very low concentrations of ammonia vapor at a sensor temperature of $80{ }^{\circ} \mathrm{C}$. Due to the high affinity of polyaniline to ammonia and presence of conductive pathways in carbon black through the composite, the sensor exhibits sensitive and fast response down to ppb concentrations. The high sensitivity, rapid response and good reversibility of the sensor make it promising for real-time ammonia sensing applications.
\end{abstract}

Key words:Polyaniline, flexible sensors, ammonia detection, solution processability, conductive composite

\section{Introduction}

Many gas sensors use electrically conducting polymers (CPs) as the sensitive layer because of their great design flexibility, ease of fabrication, low-cost, low-power consumption and tunability [1]. One of the most attractive properties of conducting polymers such as polyaniline (PANI), useful for gas sensing applications, is their reversible doping behaviour through acid/base interactions. PANI is known to be an excellent sensing material for ammonia, since $\mathrm{NH}_{3}$ deprotonates the amine group in emeraldine salt (doped form of PANI), converting it to emeraldine base (undoped form) which may cause a significant decrease in conductivity. This process is reversible in nature; however the very high affinity of PANI to ammonia has its drawbacks, mainly with regard to sensor recovery where long timescales are required to return the sensor to its baseline. Operation at elevated temperatures can improve desorption of $\mathrm{NH}_{3}$ molecules from the sensor film, hence improving the recovery time [2]. In order to make thin sensitive layers, PANI in many different forms, such as nanofibers, and composites of PANI with polymers and inorganic additives have been synthesized and some of them have shown very promising sensing properties [2].

Unfortunately, lack of solution processability, the inherent problem with conventional PANI especially in its doped state [2,3], has limited implementation of this material in conventional sensor fabrication methods. Great efforts have been made to enhance the solubility and processability of emeraldine salt. The methods that have been used fall into two main categories: (a) synthetic methods such as preparation of PANI nanoemulsions, templateguided synthesis of polyelectrolyte:PANI complexes, self-doping by graft polymerization, PANI copolymerization, etc., and (b) solution blending based on 'counter-ion induced processability' concept [4].<smiles>O=C(O)CC(C(=O)O)S(=O)(=O)O</smiles>

: acid

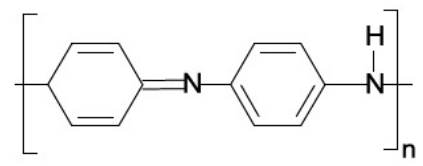

ise
Here, we have exploited sulphosuccinic acid (SSA solution, $70 \mathrm{wt} \%$ in water, SigmaAldrich), a multifunctional dopant with sulphonic and carboxylic acid groups, which not only renders the doped PANI soluble in aprotic solvents such as n-methyl pyrrolidone (NMP), but also makes it possible to prepare conductive composites of PANI/carbon black (CB) simply by mixing surface modified carbon black (20 wt.\% polyaniline on carbon black composite, Sigma-Aldrich) with PANI solution. The PANI used here was emeraldine base, average $M_{W} \sim 10,000$ (Sigma- Aldrich). Sensors were fabricated by depositing a thin layer of 


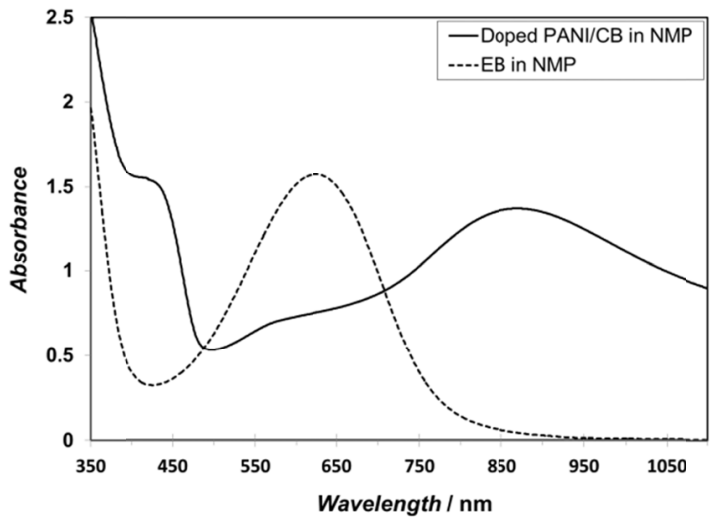

Fig.1. UV/Vis spectra of undoped and doped form of polyaniline solutions used in this study.

PANI/CB dispersion in NMP via dip coating on flexible polyimide substrates with interdigitated electrodes, incorporating a heater.

\section{Results \& Discussion}

Fig. 1 compares the UV/Vis spectrum of SSAdoped PANI/CB dispersion in NMP to that of emeraldine base. For the EB the absorption band at ca.635 nm is related to the $n-\pi^{*}$ transition (quinoid form structure). For doped polyaniline, the absorption at ca. $425 \mathrm{~nm}$ is due to the presence of localized semi quinone population or the polaron absorption, and the absorption at ca.850 $\mathrm{nm}$ due to the delocalized free electron states [3]. Furthermore, a long free tail extended to near IR region is observed for doped PANI/CB solution which may be attributed to the highly-delocalized free electron state of polyaniline doped with this multi-

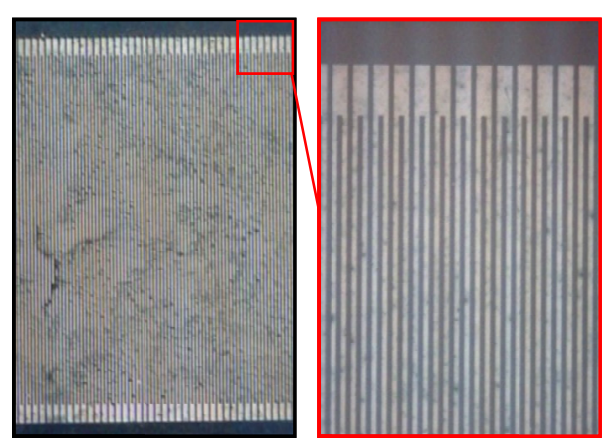

Fig. 2. Optical microscopy of sensors (The electrode gap size is $20 \mu \mathrm{m}$ ).

functional dopant. Fig. 2 shows the optical microscope images of deposited composite film on a flexible polyimide substrate with

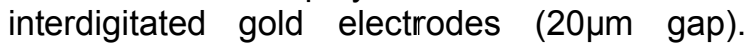
Carbon particles of max $20 \mu \mathrm{m}$ in diameter are evenly dispersed in the composite, without using vigorous mixing methods such as ultrasonication, which gives a very low resistance to the sensor (average resistance of the film is $80 \mathrm{Ohms}$ ).

Responses of the sensor held at $80{ }^{\circ} \mathrm{C}$ toward an ascending sequence of very low ammonia concentrations generated by a permeation tube (Fine Permeation Tubes, Italy) is shown in Fig. 3. The sensor response recovers in minutes, compared to hours for a sensor working at ambient temperature, for the entire range of concentrations tested. The inset of Fig. 3 shows the result of gives enough time to sensors let them recover back completely to the baseline. The sensitivity and repeatability of sensor response to ammonia ina wide concentration

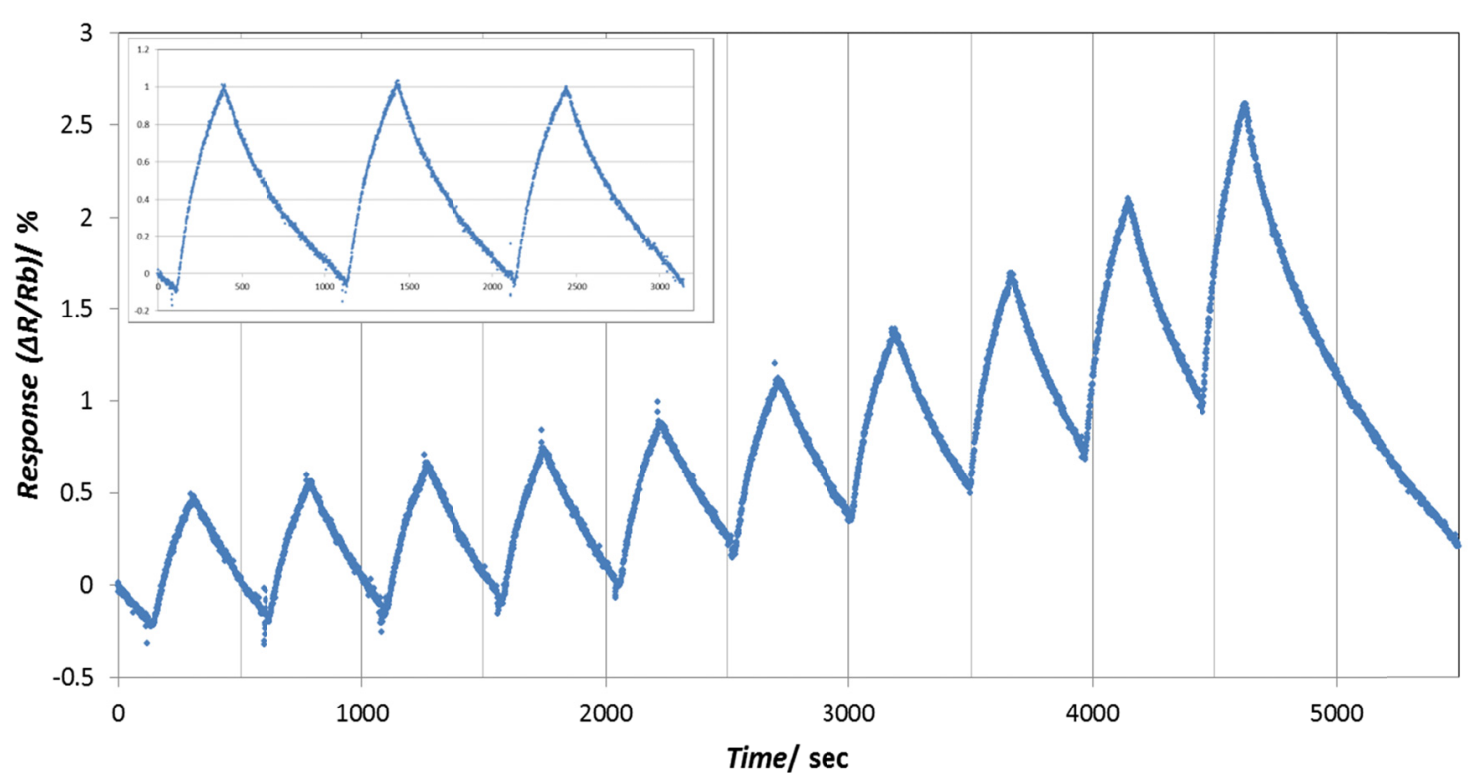

Fig. 3. Sensor response toward an ascending sequence of ammonia concentrations (425, 550, 680, 720, $850,975,1150,1360,1700,2250 \mathrm{ppb}$ ) (exposure time=3 min, recovery time $=5 \mathrm{~min}$ ). The sensor was kept at $80{ }^{\circ} \mathrm{C}$ using a subminiature heater. There is a slight baseline drift over exposure time. Inset shows reproducibility of response toward $550 \mathrm{ppb}$ ammonia (exposure time $=5 \mathrm{~min}$, recovery time=12 $\mathrm{min}$ ). 


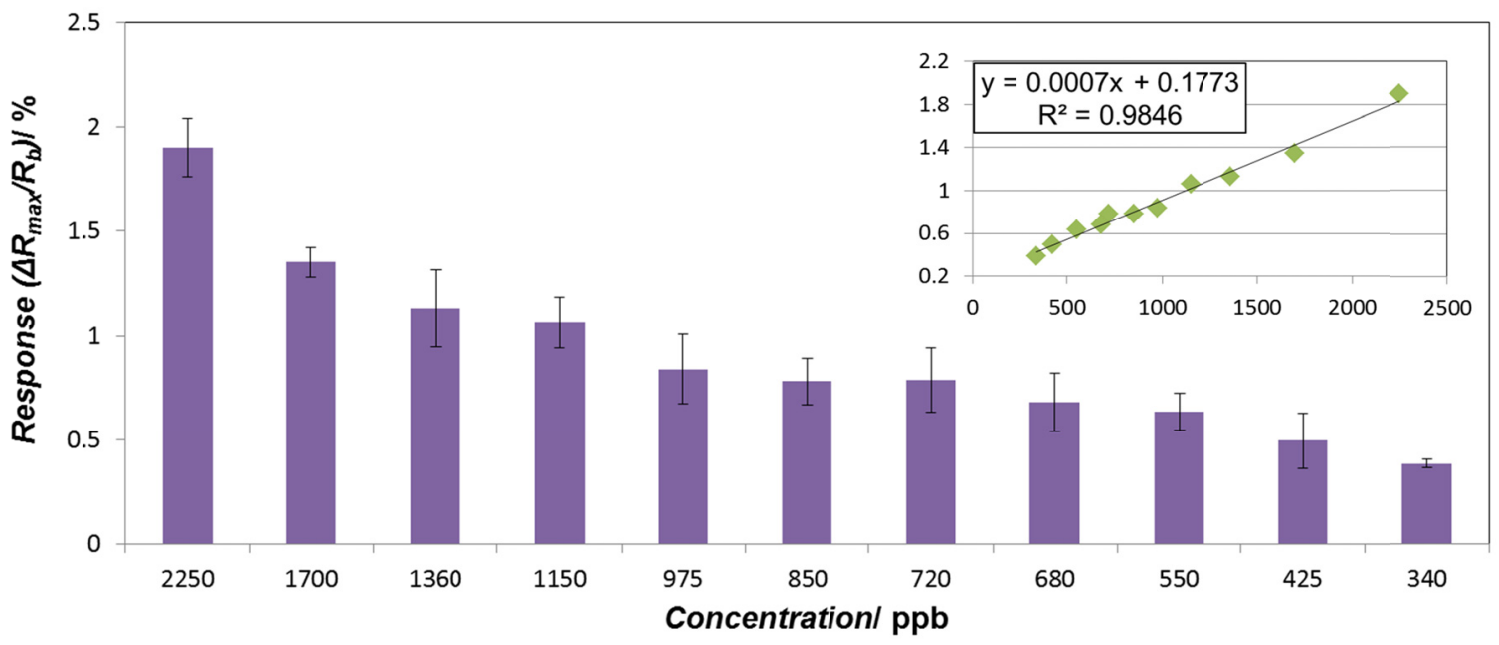

Fig. 4. Average response of the sensors calculated based on the maximum response toward both ascending and descending series of ammonia concentrations. Error bars are representative of the standard deviation of maximum sensor response at each concentration. Inset shows the linearity of response over concentration range studied here (exposure time $=3 \mathrm{~min}, T_{\text {sensor }}=80^{\circ} \mathrm{C}$ ).

range has been assessed in Fig. 4. The high sensitivity of the sensor is attributed to: (a) dedoping of the emeraldine salt and (b) disruption of carbon black conductive pathways throughout the sensing layer, upon absorption of ammonia molecules. These two simultaneous phenomena are believed to act in a synergistic way, decreasing the electrical conductance of the composite layer [5].The calibration curve shows the linearity of the response (inset of Fig. 4). Based on the data in Fig. 4, we can estimate the limit-of-detection (LOD) of the sensors using following equation:

$$
y_{L O D}=a+3 S_{y / x}
$$

Which $\mathbf{y}_{\text {OLD }}$ is the sensor response intensity at theoretical limit-of-detection, $\mathbf{a}$ is intercept of calibration curve (here: 0.1773 ) and $\frac{\mathrm{S} \mathbf{y}}{\mathrm{x}}$ is the Sy

standard deviation of the response. $\mathbf{x}$ can be calculated based on eq. (2):

$S_{y / x}=\sqrt{\frac{\left(y_{i}-\hat{y}_{i}\right)^{2}}{n-2}}$

here, $y_{i}$ is the response measured at each concentration, $\hat{y}_{i}$ is the response value determined from the calibration curve at that specific concentration, and $n$ is the number of data. Based on eq. (1), (2) and the calibration equation (inset of Fig. 4), the LOD of the sensor is calculated as $535 \mathrm{ppb}$. We believe that by increasing the data points and optimizing the sensing measurement, we can reach much lower limit-of-detection.

These results indicate a highly sensitive and rapid ammonia sensor with linear and reproducible response. These important sensing parameters make this flexible SSAdoped PANI/CB conducting composite sensor an attractive candidate for real-time ammonia sensing applications.

\section{Acknowledgements}

This work was supported by FlexSmell-FP7PEOPLE-ITN-2008-238454.

\section{References}

[1] J. Janata \& M. Josowicz, Conducting polymers in electronic chemical sensors, Nature Materials 2, pp. 19-24 (2003); doi: 10.1038/nmat768

[2] K. Crowley et al., Fabrication of an ammonia gas sensor using inkjet-printed polyaniline nanoparticles, Talanta77, 710-717 (2008); doi: 10.1016/j.talanta.2008.07.022

[3] K. Tzou, R. V. Gregory, A method to prepare soluble polyaniline salt solutions - In situ doping of PANI base with organic dopants in polar solvents, Synthetic Metals 53, 365-377 (1993); doi: 10.1016/0379-6779(93)91106-C

[4] Y. Cao, P. Smith, A. J. Heeger, Counter-ion induced processability of conducting polyaniline and of conducting polybllends of polyaniline in bulk polymers, Synthetic Metals 48, 91-97 (1992); doi: 10.1016/0379-6779(92)90053-L

[5] G. A. Sotzing et al., Highly sensitive detection and discrimination of biogenic amines utilizing arrays of polyaniline/carbon black composite vapour detectors, Chemistry of Materials 12, 593595 (2000); doi: 10.1021/cm990694e 